\title{
Suscetibilidade antimicrobiana in vitro dos Staphylococcus coagulase negativa oculares
}

\author{
In vitro antimicrobial susceptibility of coagulase negative \\ staphylococcal ocular isolates
}

\author{
Maria de Fátima Azevedo Gayoso ${ }^{1}$ \\ Adália Dias Dourado Oliveira ${ }^{2}$ \\ Pedro Alves d'Azevedo ${ }^{3}$ \\ Maria Cecília Zorat $\mathrm{Yu}^{4}$ \\ Ana Luisa Höfling-Lima ${ }^{5}$ \\ Waldemar Francisco ${ }^{6}$
}

\section{RESUMO}

Objetivo: Avaliar a suscetibilidade, in vitro, de Staphylococcus coagulase negativa $(\mathrm{SCoN})$, isolados da conjuntiva e córnea, à meticilina, às fluoroquinolonas e aos aminoglicosídeos. Métodos: Foram analisadas retrospectivamente 707 amostras oculares de SCoN quanto à suscetibilidade aos antimicrobianos pelo teste de disco difusão, durante o período de janeiro de 2000 a dezembro de 2003. Resultados: Houve um aumento do número de SCoN em isolados da conjuntiva $(n=57$, ano de 2000 e $n=153$, ano de 2003) e da córnea ( $n=28$, ano de 2000 e $n=78$, ano de 2003). A freqüência de $\mathrm{SCoN}$ resistentes à meticilina isolados da conjuntiva $\mathrm{e}$ da córnea, aumentou (1,8 a 19,6\% e 14,3 a 29,3\% respectivamente) durante o período avaliado. Não houve diferença estatisticamente significante nos anos estudados, nos percentuais de $\mathrm{SCoN}$ resistentes às fluoroquinolonas, nas conjuntivas (ofloxacina: 1,8 a 7,8\% e ciprofloxacina: 3,5 a 9,2\%) e nas córneas (ofloxacina: 14,3 a 9,0\% e ciprofloxacina: 14,3 a 10,3\%). Avaliando-se os resultados das amostras isoladas da conjuntiva, observou-se um aumento na resistência à tobramicina: 15,8 a 34,6\%; e à gentamicina: 10,5 a 25,5\%; mas não houve mudança no perfil de resistência das amostras da córnea à tobramicina: 28,6 a 26,9\% e à gentamicina: 21,4 a 23,1\%). Conclusão: Houve diminuição na suscetibilidade in vitro dos SCoN para meticilina, tobramicina e gentamicina. As fluoroquinolonas, representadas pela ofloxacina e ciprofloxacina, demonstraram ter um padrão estável de suscetibilidade in vitro.

Descritores: Resistência microbiana a drogas; Infecções oculares bacterianas/microbiologia; Staphylococcus/isolamento \& purificação; Coagulase; Fluoroquinolonas; Aminoglicosídeos; Resistência a meticilina; Conjuntivites; Ceratites

Trabalho realizado no Departamento de Oftalmologia da Universidade Federal de São Paulo - UNIFESP.

Pós-graduanda da Universidade Federal de São Paulo UNIFESP - São Paulo (SP) - Brasil.

2 Pós-graduanda da UNIFESP - São Paulo (SP) - Brasil.

${ }^{3}$ Pós-doutorando da UNIFESP - São Paulo (SP) - Brasil.

${ }^{4}$ Biomédica responsável pelo Laboratório de Microbiologia do Departamento de Oftalmologia da UNIFESP São Paulo (SP) - Brasil.

${ }^{5}$ Livre-docente, Professora titular do Departamento de Oftalmologia da UNIFESP - São Paulo (SP) - Brasil.

${ }^{6}$ Doutor, Professor Assistente do Departamento de Microbiologia do Instituto de Ciências Biológicas (ICB) da Universidade de São Paulo - USP - São Paulo (SP) Brasil.

Endereço para correspondência: Av. Ibijaú, 331 - 17ํำ andar - São Paulo (SP) CEP 04524-020

E-mail: fatimagayoso@hotmail.com

Recebido para publicação em 25.09.2006

Última versão recebida em 21.05.2007

Aprovação em 22.08.2007

\begin{abstract}
INTRODUÇÃO
Infecções bacterianas oculares, como ceratites, conjuntivites e endoftalmites, podem evoluir com graves conseqüências para o olho e conseqüentemente para a manutenção da visão, principalmente se o tratamento apropriado com antimicrobianos for tardio ${ }^{(1-2)}$.

$\mathrm{Na}$ conduta de atenção oftalmológica a estas infecções, os tratamentos são freqüentemente iniciados antes da identificação do patógeno causador e dos resultados dos testes de suscetibilidade aos antimicrobianos estarem disponíveis. A escolha dos antimicrobianos recai então sobre drogas que historicamente demonstram efetividade in vivo e in vitro, contra um amplo espectro de possíveis microrganismos. Cada vez mais isolam-se microrganismos resistentes aos fármacos mais comumente utilizados ${ }^{(3)}$.
\end{abstract}

No inicio dos anos 40, quando a penicilina foi lançada com sucesso 
para tratamento das infecções, Staphylococcus spp eram quase $90 \%$ suscetíveis a esta droga. Logo, desenvolveu-se a resistência bacteriana à penicilina natural e foi acompanhada pela resistência às penicilinas semi-sintéticas ${ }^{(4-6)}$. Desde então, o aparecimento de amostras de Staphylococus coagulase negativa $(\mathrm{SCoN})$ meticilina resistente têm aumentado significativamente no mundo inteiro ${ }^{(7-8)}$.

A resistência bacteriana à meticilina, deve-se, na maioria das vezes, à modificação de uma proteína adicional de ligação à penicilina $\left(\mathrm{PBP}_{2 \mathrm{a}}\right)$ codificada pelo gen mec $A$. Normalmente, a resistência a esta droga está associada com resistência cruzada in vitro a outros antimicrobianos, incluindo fluoroquinolonas e aminoglicosídeos ${ }^{(6,9)}$.

\section{OBJETIVO}

O objetivo deste estudo foi avaliar o padrão de suscetibilidade, in vitro, de SCoN, isolados da conjuntiva e córnea em relação à meticilina, fluoroquinolonas (ofloxacina e ciprofloxacina) e aos aminoglicosídeos (tobramicina e gentamicina).

\section{MÉTODOS}

Foram analisadas retrospectivamente 707 amostras de SCoN isolados de conjuntivas e córneas, processadas no Laboratório de Microbiologia Ocular do Departamento de Oftalmologia da Universidade Federal de São Paulo (LOFT- Unifesp), durante o período de janeiro de 2000 a dezembro de 2003.

Todas as amostras da conjuntiva analisadas foram obtidas do fundo-de-saco da conjuntiva inferior, usando uma zaragatoa alginatada estéril, e imediatamente inoculadas em Ágar sangue de carneiro a 5\% (AS) (Difco, Le Pont de Claix/France), Ágar chocolate (AC) (Difco, Le Pont de Claix/France), Brain Heart Infusion Broth (BHI) (Difco, Le Pont de Claix/France) e/ou Tioglicolato (TIO) (Difco, Le Pont de Claix/France).

Os raspados de córnea foram realizados com espátula de Kimura e igualmente inoculados em meios de cultura AS, AC e ainda em Ágar Sabouraud (Difco/Le Pont de Claix/France) e em meios líquidos (BHI e /ou TIO).

As placas e os tubos das culturas foram incubados a $35^{\circ} \mathrm{C}$, em média por 21 dias e as colônias bacterianas isoladas foram identificadas pela morfologia, testes da catalase e Dnase (Difco, Le Pont de Claix/France) e pelas características morfológicas na coloração de Gram, realizados em esfregaços dos materiais conjuntivais e corneanos.
Os testes de suscetibilidade foram realizados com discos: de $1 \mu \mathrm{g}$ de oxacilina para meticilina (Cecon, São Paulo, Brasil), $5 \mu \mathrm{g}$ de ofloxacina (Cecon, São Paulo, Brasil), $5 \mu \mathrm{g}$ de ciprofloxacina (Cecon, São Paulo, Brasil), 10 $\mu$ g de gentamicina (Cecon, São Paulo, Brasil) e $10 \mu \mathrm{g}$ de tobramicina (Cecon, São Paulo, Brasil); utilizando-se o método de disco difusão. Os resultados foram interpretados pelos padrões do NCCLS (National Commitee for Clinical Laboratory Standarts - 2003) ${ }^{(10)}$. Os dados coletados foram submetidos à análise estatística, sendo aplicados os testes de Mann-Whitney e de Friedman.

\section{RESULTADOS}

No período analisado, 707 culturas foram positivas para o SCoN, sendo 435 isoladas de conjuntiva e 272 isoladas de córnea. $\mathrm{O}$ número de conjuntivites e ceratites causadas pelos SCoN, por ano de estudo, encontram-se na tabela 1. Os resultados evidenciam aumento crescente a cada ano (Gráfico 1).

Em relação à conjuntiva, houve uma diferença estatisticamente significante, entre os anos avaliados, com relação à proporção de amostras de conjuntiva resistentes à meticilina (oxacilina) $(\mathrm{p}<0,001)$. As proporções encontradas nos anos de $2000(1,8 \%)$ e $2001(0,9 \%)$ foram semelhantes e significantemente menores do que no ano de 2002 (9,3\%), que por sua vez se mostrou significantemente menor do que no ano de 2003 $(19,6 \%)$. Não foi encontrada diferença estatisticamente significante, em relação à resistência da ciprofloxacina $(\mathrm{p}=0,543)$ e da ofloxacina $(p=0,270)$. Para a tobramicina, existiu diferença estatisticamente significante em relação à proporção de bactérias resistentes $(p<0,001)$. As proporções encontradas nos anos de 2000 (15,8\%), $2001(9,3 \%)$ e 2002 (17,8\%) foram semelhantes e significantemente menores do que no ano de 2003 (34,6\%). Por último foi comprovado um aumento estatisticamente significante de bactérias resistentes à gentamicina $(p=0,003)$, onde as proporções encontradas nos anos de 2000 (10,5\%) e 2001 (9,3\%) foram semelhantes e significantemente menores do que no ano de 2003 (25,5\%). Para este antimicrobiano, a proporção encontrada nos ano de 2002 (17,8\%) não diferiu dos demais anos (Tabela 2 e Gráfico 2).

Quando analisamos os resultados dos materiais obtidos da córnea para os SCoN encontramos diferença estatisticamente significante entre os anos avaliados em relação à proporção de bactérias resistentes à meticilina (oxacilina) $(\mathrm{p}=0,003)$, onde $\mathrm{a}$ proporção encontrada no ano de $2001(7,7 \%)$ foi significante-

\begin{tabular}{|c|c|c|c|c|c|c|c|c|c|}
\hline & $n$ & $\%$ & $n$ & $\%$ & $\mathrm{n}$ & $\%$ & $n$ & $\%$ & $\frac{\text { Total }}{n}$ \\
\hline Córnea & 28 & 10,3 & 78 & 28,7 & 88 & 32,4 & 78 & 28,7 & 272 \\
\hline
\end{tabular}


mente menor do que no ano de 2003 (29,5\%). As proporções encontradas nos anos de $2000(14,3 \%)$ e 2002 (14,8\%) não diferiram entre si nem entre os demais anos. Não foi encontrada diferença estatisticamente significante para resistência aos demais antimicrobianos: ciprofloxacina $(p=0,787)$, ofloxacina $(\mathrm{p}=0,813)$, tobramicina $(\mathrm{p}=0,268)$ e gentamicina $(\mathrm{p}=0,672)$ (Tabela 3 e Gráfico 3).

\section{DISCUSSÃO}

A resistência à meticilina representa um sério problema, tanto do ponto de vista de sua detecção, como por sua importância clínica. Devemos lembrar que a resistência dos Staphylococcus ssp à meticilina implica em resistência a todos os antimicrobianos beta-lactâmicos e apresentam um padrão de resistência múltipla associada a outros antimicrobianos de uso freqüente na Oftalmologia, tais como as quinolonas e os aminoglicosídeos ${ }^{(6)}$.

Constitui ainda preocupação, o fato de que estas bactérias constituem a microbiota conjuntival de pacientes normais, candidatos a cirurgias com manipulação de tecidos intra-oculares e extra-oculares (refrativas), o que torna necessário a escolha de profilaxia pré-operatória eficiente pela possibilidade de resultados pós-operatórios desfavoráveis.

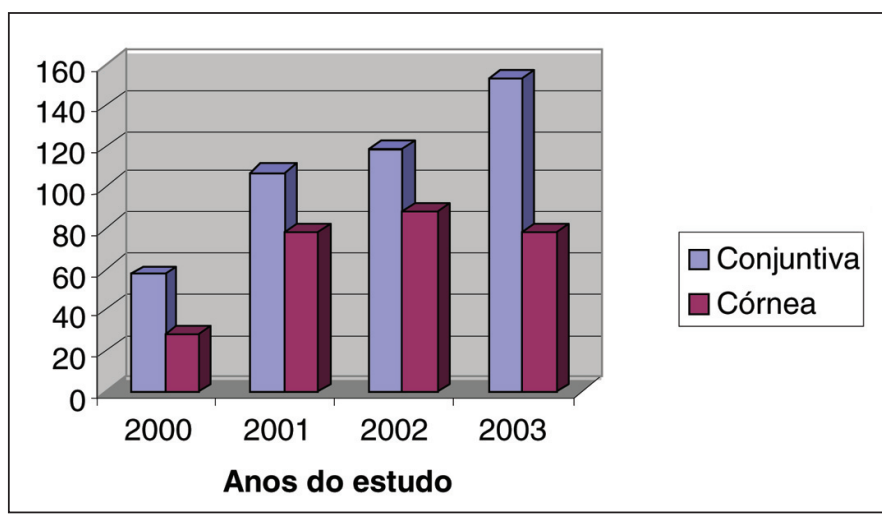

Gráfico 1 - Staphylococcus coagulase negativa isolados em conjuntivas e córneas entre janeiro de 2000 e dezembro de 2003
Diversos estudos nacionais ${ }^{(1-2,11)}$ e internacionais ${ }^{(3-9,12-17)}$ foram realizados, buscando avaliar não somente a etiologia como também a eficácia clínica e a atividade de diferentes antimicrobianos para as doenças oculares, ressaltando como agentes os Staphylococcus ssp.

Em 1998, foi demonstrada resistência aumentada à gentamicina e meticilina ${ }^{(9)}$, e neste mesmo ano, outra pesquisa concluiu que as fluoroquinolonas eram superiores aos aminoglicosídeos no tratamento das infecções oculares pelo SCoN, estudando a suscetibilidade antimicrobiana de amostras oculares isoladas em 12 laboratórios da América do Norte e América do Sul ${ }^{(3)}$.

Na nossa comunidade, foi relatada resistência já estabelecida $^{(2)}$, à meticilina e aos aminoglicosídeos e uma tendência à resistência às fluoroquinolonas (ciprofloxacina e ofloxacina), ao descrever o padrão de suscetibilidade in vitro de bactérias oculares aos mais diversos antimicrobianos, em um período de 15 anos no LOFT-Unifesp-EPM (1985 a 2000).

Em $2002^{(13)}$ e em 2004(1), também foi demonstrada, uma

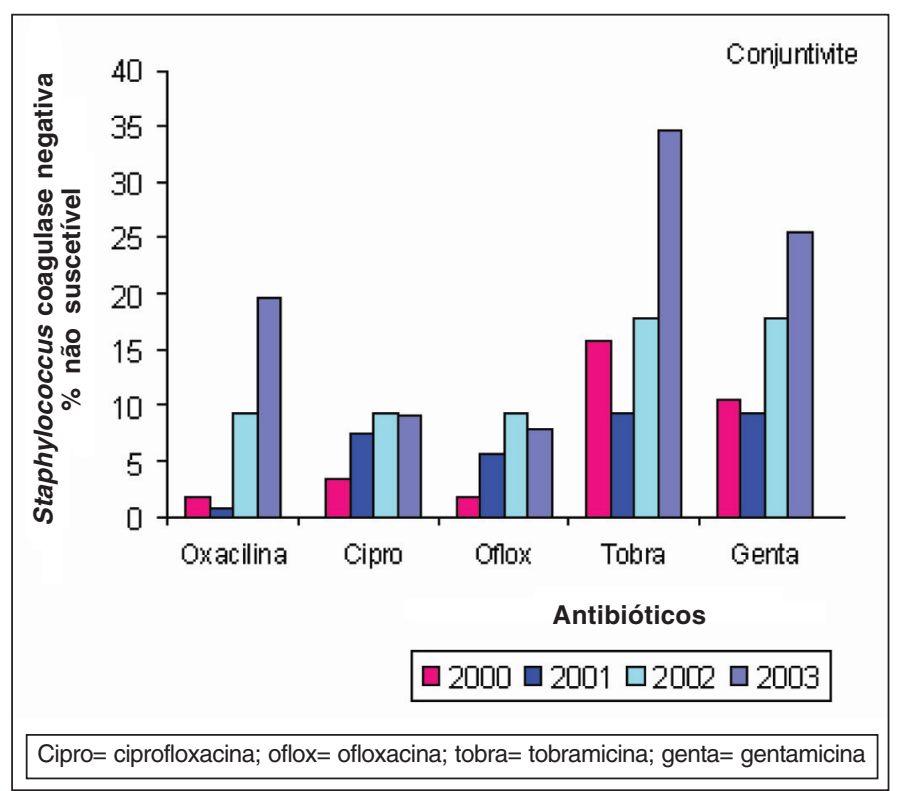

Gráfico 2 - Perfil de resistência dos SCoN isolados de conjuntivas

\begin{tabular}{|c|c|c|c|c|c|c|c|c|c|}
\hline & \multicolumn{2}{|c|}{2000} & \multicolumn{2}{|c|}{2001} & \multicolumn{2}{|c|}{2002} & \multicolumn{2}{|c|}{2003} & \multirow{2}{*}{ Qui-quadrado } \\
\hline & $\bar{n}$ & $\%$ & $\mathbf{n}$ & $\%$ & $\mathbf{n}$ & $\%$ & $\mathbf{n}$ & $\%$ & \\
\hline Resistência aos antimicrobianos & 57 & & 107 & & 118 & & 153 & & \\
\hline Meticilina (oxacilina) & 1 & 1,8 & 1 & 0,9 & 11 & 9,3 & 30 & 19,6 & $\begin{array}{l}\mathrm{p}<0,001^{*} \\
(2000=2001)<2002<2003\end{array}$ \\
\hline Ciprofloxacina & 2 & 3,5 & 8 & 7,5 & 11 & 9,3 & 14 & 9,2 & $p=0,543$ \\
\hline Ofloxacina & 1 & 1,8 & 6 & 5,6 & 11 & 9,3 & 12 & 7,8 & $p=0,270$ \\
\hline Gentamicina & 6 & 10,5 & 10 & 9,3 & 21 & 17,8 & 39 & 25,5 & $\begin{array}{l}\mathrm{p}=0,003^{*} \\
(2000=2001)<2003\end{array}$ \\
\hline
\end{tabular}




\begin{tabular}{|c|c|c|c|c|c|c|c|c|c|}
\hline & \multicolumn{2}{|c|}{2000} & \multicolumn{2}{|c|}{2001} & \multicolumn{2}{|c|}{2002} & \multicolumn{2}{|c|}{2003} & \multirow[t]{2}{*}{ Qui-quadrado } \\
\hline & $\bar{n}$ & $\%$ & $\mathrm{n}$ & $\%$ & $\mathbf{n}$ & $\%$ & $\mathbf{n}$ & $\%$ & \\
\hline Resistência aos antimicrobianos & 28 & & 78 & & 88 & & 78 & & \\
\hline Ciprofloxacina & 4 & 14,3 & 7 & 9,0 & 7 & 8,0 & 8 & 10,3 & $p=0,787$ \\
\hline Ofloxacina & 4 & 14,3 & 10 & 12,8 & 9 & 10,2 & 7 & 9,0 & $p=0,813$ \\
\hline Gentamicina & 6 & 21,4 & 12 & 15,4 & 17 & 19,3 & 18 & 23,2 & $p=0,672$ \\
\hline
\end{tabular}

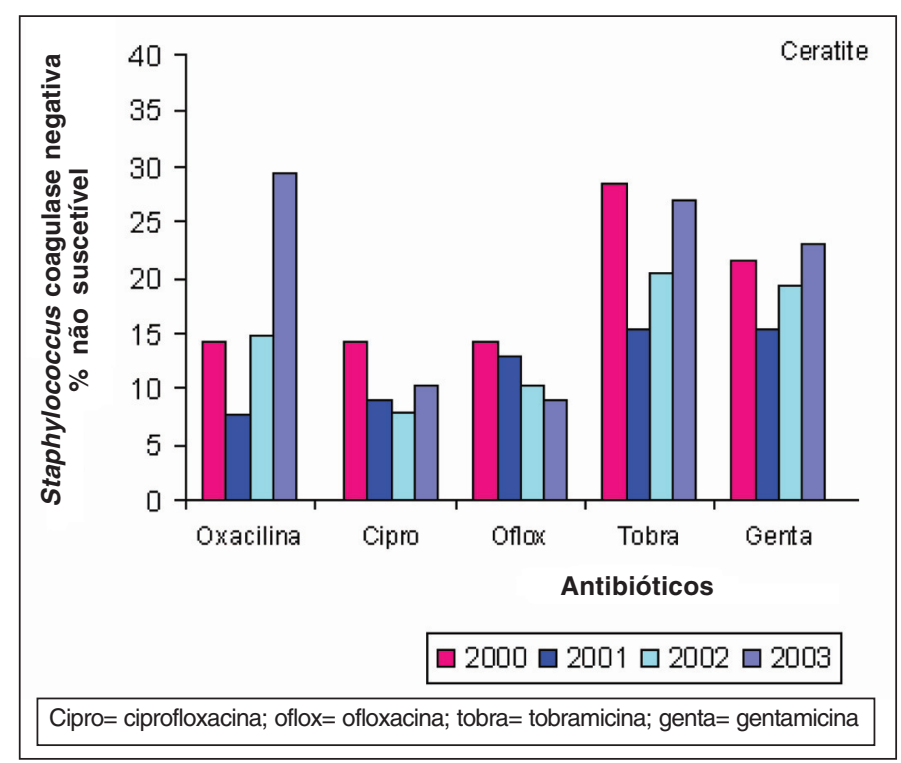

Gráfico 3 - Perfil de resistência dos SCoN isolados de córneas

tendência de resistência às fluoroquinolonas de segunda geração e maior eficácia das fluoroquinolonas de quarta geração ${ }^{(1,13)}$.

Este estudo procura dar seguimento à análise dos padrões de resistência dos SCoN isolados de conjuntiva e córnea dos pacientes do LOFT-Unifesp-EPM nos quatro anos subseqüentes, uma vez que este microrganismo é comprovadamente o mais freqüentemente isolado em amostras oculares.

Embora os valores de MIC (Minimal Inhibitory Concentration) sejam importantes indicadores in vitro da atividade ou potência dos antimicrobianos, estes não necessariamente predizem a eficácia in vivo. A eficácia clínica depende da concentração inibitória mínima atingida no tecido deseja$\mathrm{do}^{(18)}$. Sendo assim, torna-se necessário comparar resultados in vitro com vários parâmetros farmacológicos para compreensão plena e escolha dos agentes antimicrobianos e de seu potencial terapêutico verdadeiro. Principalmente, por se tratar do olho o órgão alvo, é importante considerar a penetração ocular na córnea, na conjuntiva e no humor aquoso, já que o MIC, refere-se à concentração sanguínea da droga.

Assim, testes de suscetibilidade devem acompanhar a avaliação laboratorial de infecções oculares visando manter o conhecimento do espectro de ação de agentes antimicrobianos em determinada área geográfica. Foi sugerido já em 1 . Estes tipos de testes devem ter seus resultados analisados e comparados retrospectivamente a cada 2 a 3 anos $^{(3)}$, para detectar tendências à resistência aos antimicrobianos de uso corrente ${ }^{(2)}$.

Desta maneira, nosso estudo revela que Staphylococcus coagulase negativa $(\mathrm{SCoN})$ é um importante patógeno responsável por vários casos de conjuntivite e ceratite nos pacientes que procuraram o serviço de Oftalmologia da UNIFESP-EPM. A maioria destes microorganismos vem apresentando aumento na resistência in vitro à meticilina e aos aminoglicosideos estudados (tobramicina e gentamicina). As fluoroquinolonas, representadas neste estudo pela ofloxacina e ciprofloxacina, permaneceram como uma boa escolha para o tratamento destas bactérias durante o período estudado. Entretanto, já demonstram uma tendência ao desenvolvimento de resistência, o que causa grande preocupação pois restringe as opções de tratamento e conduta destas corriqueiras infecções oculares.

\section{ABSTRACT}

Purpose: To assess the in vitro susceptibility of conjunctival and corneal coagulase negative Staphylococcus (CoNS) to methicillin, fluoroquinolones and aminoglycosides. Methods: A total of 707 conjunctival and corneal CoNS disk diffusion test results were retrospectively analyzed, from January 2000 through December 2003. Results: From 2000 to 2003, there was an increase in number of CoNS isolated from conjunctiva $(n=57$ to $n=153)$ and cornea $(n=28$ to $n=78)$. The frequency of conjunctival and corneal methicillin-resistant CoNS also increased (1.8 to $19.6 \%$ and 14.3 to $29.3 \%$, respectively). There was no statistically significant difference between fluoroquinolones-resistant CoNS percentages in conjunctiva (ofloxacin: 1.8 to $7.8 \%$ and ciprofloxacin: 3.5 to $9.2 \%$ ) and cornea (ofloxacin: 14.3 to $9.0 \%$ and ciprofloxacin: 14.3 to $10.3 \%)$. Evaluating the results of the conjunctival samples, there was increased resistance to tobramycin (15.8 to $34.0 \%)$ and to gentamycin (10.5 to $25.5 \%$ ). There was no change in resistance of corneal isolates to tobramycin (28.6 to $26.9 \%$ ) 
and to gentamycin (21.4 to 23.1\%). Conclusions: there was a decrease in in vitro CoNS susceptibility to methicillin, tobramycin and gentamycin. Fuoroquinolones represented by ofloxacin and ciprofloxacin demonstrated stable in vitro susceptibility.

Keywords: Drug resistance, microbial; Eye infections, bacte$\mathrm{rial} /$ microbiology; Staphylococcus/isolation \& purification; Coagulase; Fluoroquinolones; Aminoglycosides; Methicillin resistance; Conjunctivitis; Keratitis.

\section{REFERÊNCIAS}

1. Höfling-Lima AL, Belfort Júnior R, Moeller CTA, Castelo Branco B, Sousa LB, Freitas D. In vitro antibiotic susceptibilities of ocular bacteria isolates from the cornea and conjunctiva to moxifloxacin, gatifloxacin and other fluoroquinolones. Arq Bras Oftalmol. 2004;67(6):883-6.

2. Chalita MR, Höfling-Lima AL, Paranhos A Jr, Schor P, Belfort R Jr. Shifting trends in in vitro antibiotic susceptibilities for common ocular isolates during a period of 15 years. Am J Ophthalmol. 2004;137(1):43-51.

3. Jensen HG, Felix C. In vitro antibiotic susceptibilities of ocular isolates in North and South America. In Vitro Antibiotic Testing Group. Cornea. 1998; 17(1):79-87. Comment in: Cornea. 1998;17(5):571-2.

4. Fukuda M, Ohashi H, Matsumoto C, Mishima S, Shimomura Y. Methicillin-resistant Staphylococcus aureus and methicillin-resistant coagulase-negative Staphylococcus ocular surface infection efficacy of chloramphenicol eye drops. Cornea. 2002;21(7 Suppl):S 86-9.

5. Gil de M M. Staphylococcus aureus: Microbiologia y aspectos moleculares de la resistencia a meticilina. Rev Chil Infectol. 2000;17(2):145-52.

6. Marin M. Resistencia del Staphylococcus a la meticilina. Medicina (B Aires). 2002;62(Supl 2):30-5

7. Alexandrakis G, Alfonso EC, Miller D. Shifting trends in bacterial keratitis in south Florida and emerging resistance to fluoroquinolones. Ophthalmology. 2000;107(8):1497-502.

8. Kato T, Hayasaka S. Methicillin-resistant Staphylococcus aureus and methicillin-resistant coagulase-negative staphylococci from conjunctivas of preoperative patients. Jpn J Ophthalmol. 1998;42(6):461-5.

9. Davis JL, Koidou-Tsiligianni A, Pflugfelder SC, Miller D, Flynn HW Jr, Forster RK. Coagulase-negative staphylococcal endophthalmitis. Increase in antimicrobial resistance. Ophthalmology. 1988;95(10):1404-10.

10. National Committee for Clinical Laboratory Standards (NCCLS). 2004. Performance standards for antimicrobial susceptibility testing; Fourteenth information supplement: M100-S14. NCCLS, Villanova, PA. 2004.

11. Leal F, Höfling-Lima AL, Freitas D, Campos M. Análise laboratorial das ceratites infecciosas secundárias à cirurgia refrativa. Arq Bras Oftalmol. 2005; 68(3):353-6.

12. Goldstein MH, Kowalski RP, Gordon YJ. Emerging fluoroquinolone resistance in bacterial keratitis: a 5-year review. Ophthalmology. 1999;106(7): 1313-8.

13. Mather R, Karenchak LM, Romanowski EG, Kowalski RP. Fourth generation fluoroquinolones: new weapons in the arsenal of ophthalmic antibiotics. Am J Ophthalmol. 2002;133(4):463-6.

14. Miño de Kaspar H, Koss MJ, He L, Blumenkranz MS, Ta CN. Antibiotic susceptibility of preoperative normal conjunctival bacteria. Am J Ophthalmol. 2005;139(4):730-3.

15. Recchia FM, Busbee BG, Pearlman RB, Carvalho-Recchia CA, Ho AC. Changing trends in the microbiologic aspects of postcataract endophthalmitis. Arch Ophthalmol. 2005;123(3):341-6.

16. Ta CN, Chang RT, Singh K, Egbert PR, Shriver EM, Blumenkranz MS, Miño de Kaspar H. Antibiotic resistance patterns of ocular bacterial flora: a prospective study of patients undergoing anterior segment surgery. Ophthalmology. 2003;110(10):1946-51.

17. Tungsiripat T, Sarayba MA, Kaufman MB, Sweet PM, Taban M, Carpenter TR, McDonnell PJ. Fluoroquinolone therapy in multiple-drug resistant staphylococcal keratitis after lamellar keratectomy in a rabbit model. Am J Ophthalmol. 2003;136(1):76-81.

18. Liu P, Müller M, Derendorf H. Rational dosing of antibiotics: the use of plasma concentrations versus tissue concentrations. Int J Antimicrob Agents. 2002;19(4):285-90. 